\title{
A Multi-laboratory in Vitro Study to Compare Data from Abbreviated and Pharmacopeial Impactor Measurements for Orally Inhaled Products: a Report of the European Aerosol Group (EPAG)
}

\author{
Steven C. Nichols, ${ }^{1}$ Jolyon P. Mitchell, ${ }^{2,8}$ Dennis Sandell, ${ }^{3}$ Patrik U. Andersson, ${ }^{4}$ Manfred Fischer, ${ }^{5}$ \\ Markus Howald, ${ }^{5}$ Roy Pengilley, ${ }^{6}$ and Phillip Krüger ${ }^{7}$
}

Received 4 November 2015; accepted 21 December 2015; published online 13 January 2016

\begin{abstract}
Fine particle dose (FPD) is a critical quality attribute for orally inhaled products (OIPs). The abbreviated impactor measurement (AIM) concept simplifies its measurement, provided there is a validated understanding of the relationship with the full resolution pharmacopoeial impactor (PIM) data for a given product. This multi-center study compared fine particle dose determined using AIM and PIM for five dry powder inhaler (DPIs) and two pressurized metered-dose inhaler (pMDI) products, one of which included a valved holding chamber (VHC). Reference measurements of FPD PIM were made by each organization using either the full-resolution Andersen 8-stage non-viable impactor (ACI) or Next

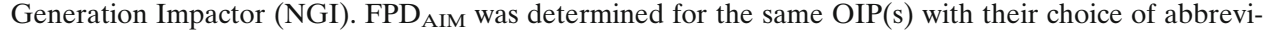
ated impactor (fast screening impactor (FSI), fast screening Andersen (FSA), or reduced NGI (rNGI)). Each organization used its validated assay method(s) for the active pharmaceutical ingredient(s) (APIs) involved. Ten replicate measurements were made by each procedure. The upper size limit for FPD AIM $_{\text {II }}$ varied from 4.4 to $5.0 \mu \mathrm{m}$ aerodynamic diameter, depending upon flow rate and AIM apparatus; the corresponding size limit for FPD $_{\text {PIM }}$ was fixed at $5 \mu \mathrm{m}$ in accordance with the European Pharmacopoeia. The $90 \%$ confidence interval for the ratio $\left[\mathrm{FPD}_{\mathrm{AIM}} / \mathrm{FPD}_{\mathrm{PIM}}\right]$, expressed as a percentage, was contained in the predetermined $85-118 \%$ acceptance interval for nine of the ten comparisons of FPD. The average value of this ratio was $105 \%$ across all OIPs and apparatuses. The findings from this investigation support the equivalence of AIM and PIM for determination of FPD across a wide range of OIP platforms and measurement techniques.
\end{abstract}

KEY WORDS: abbreviated impactor; aerosol measurement; cascade impactor; fine particle dose; inhaler.

\section{INTRODUCTION}

The aerodynamic particle size distribution (APSD) of aerosols emitted by actuation of a pressurized metered dose inhaler (pMDI) or a dry powder inhaler (DPI) is a critical quality attribute because aerodynamic size (diameter) is closely linked with, but not predictive of, the location of particle deposition in the human respiratory tract (1). Aerodynamic diameter is, therefore, associated with the physiological action of the emitted active pharmaceutical ingredient (API), or APIs in the case of a combination product, either at receptor sites along the airways pertinent to the drug class being delivered for topical

${ }^{1}$ OINDP Consultant, Rugby, Warwickshire, UK.

${ }^{2}$ Jolyon Mitchell Inhaler Consulting Services Inc., 1154 St. Anthony Road, London, Ontario, CanadaN6H 2R1.

${ }^{3}$ S5 Consulting, Ekvägen 8, Blentarp, Sweden.

${ }^{4}$ AstraZeneca, Gothenburg, Sweden.

${ }^{5}$ SkyePharma, Muttenz, Switzerland.

${ }^{6}$ Zentiva Inhalationprodukte, Munich, Germany.

${ }^{7}$ Sofotec GmbH, Bad Homburg, Germany.

${ }^{8}$ To whom correspondence should be addressed. (e-mail: mitchelljolyon@gmail.com) administration (2) or at the gas exchange region associated with the alveolar sacs in the case of systemic delivery (3). It follows that accurate and precise measures of properties related to aerodynamic diameter are key goals of methods developed to assess the size-related properties of orally inhaled product (OIP)-generated aerosols during the product life-cycle (4). The traditional procedures given in the pharmacopeial compendia involve the use of multi-stage cascade impactors $(5,6)$, which, though fulfilling the requirements for method suitability, are laborious to use and are also prone to operator errors (7), often resulting in lengthy investigations for measures that often appear to be out-of-specification, rather than due to a product batch of unacceptable quality (8). The development of the Next Generation Impactor (NGI) in the early 2000s was intended to simplify the measurement procedure by avoiding the need to assemble and take apart each stage for every determination (9). However, like all full resolution cascade impactors, there is still the need to make numerous assays of (each) API in the formulation being evaluated in order to determine the fine particle dose (FPD). However, APSDs may contain more information that may be required for the purpose of assessing product quality (10). 
The abbreviated impactor measurement (AIM) approach was developed out of a need to simplify the process of determining the fine particle portion of the dose (10), set at $5 \mu \mathrm{m}$ aerodynamic diameter in the European Pharmacopeia (Ph.Eur.) (5), but otherwise chosen to be close to that limit based on the cut-point of the appropriate size fractionating stage of the cascade impactor (CI). This concept, adapted for the purpose of demonstrating OIP quality, involves the elimination of all but one size-fractionating stage of the cascade impactor and retention of a final filter. The non-sizing components (USP/Ph.Eur. induction port and pre-separator (if needed)) are also retained. Figure 1 illustrates key features of the AIM concept, taking the fast screening Andersen impactor (FSA, Copley Scientific Ltd, Nottingham, UK) as an example of an abbreviated apparatus. Detailed descriptions of the features of such AIM-based apparatuses have been published previously (11). A survey undertaken by EPAG in 2012 to gather an industry-wide perspective on the adoption of the AIM concept revealed that the majority of the 12 organizations who responded were increasing their use of AIM-based techniques particularly for early stage development (12). All organizations were continuing to use their full resolution impactors (either Andersen 8-stage non-viable Cascade Impactor (ACI) or NGI) for instances in which complete APSDs are required in support of OIP quality assessment and control (12).

The purpose of the present experimental investigation, commissioned and coordinated by the European Pharmaceutical Aerosol Group (EPAG), was to increase the understanding of the relationships between measurements undertaken using AIM-based methodologies in relation to the pharmacopeial procedures for APSD determination using full resolution CI. The use of stage groupings as a method to effect data reduction from full resolution multi-stage CIs has the potential for introducing sampling error associated with summing data obtained from different stages, especially where the mass collected on some stages approaches the lower limit of quantitation (4). An AIM-based procedure avoids this issue by virtue that all the fine particle mass is collected on any succeeding stage (one participant, using an FSA, inserted a second stage with a cut-point of $1.1 \mu \mathrm{m}$ aerodynamic diameter following the stage whose cut-point size was at the size used to separate fine from coarse particulate), or the back-up filter (or micro-orifice collector if used in association with the NGI), in association with the single size-fractionating stage of the apparatus. Likewise, all the coarse component of the impactorsized mass is collected above the size-fractionating stage with its cut-point near to or at $5 \mu \mathrm{m}$ aerodynamic diameter. The intention was to provide a cornerstone in the validation and verification of the utility of AIM-based methodology, by utilizing a multi-center approach which included a broad spectrum of OIPs including pMDI and DPI classes of inhaler. The primary goal was to investigate whether an AIM-based apparatus could be used in place of a full resolution CI, termed pharmaceutical impactor measurement (PIM), to acquire equivalent values for FPD as the principal quality measurement for an orally inhaled product. Secondary goals were to compare other important measures related to the performance of the test inhaler, in particular (total) delivered dose (DD), mass of medication deposited in the induction port ("throat" dose), and coarse particle dose (CPD). Therefore, it was established for each OIP that each metric was substantially equivalent to the same measure determined using a selected PIM-based full resolution apparatus. Although these goals represent the ideal situation, it is recognized that an AIM procedure might still be acceptable if it provided measures of FPD that were off-set from the corresponding PIMbased measures, provided that the cause of the off-set was

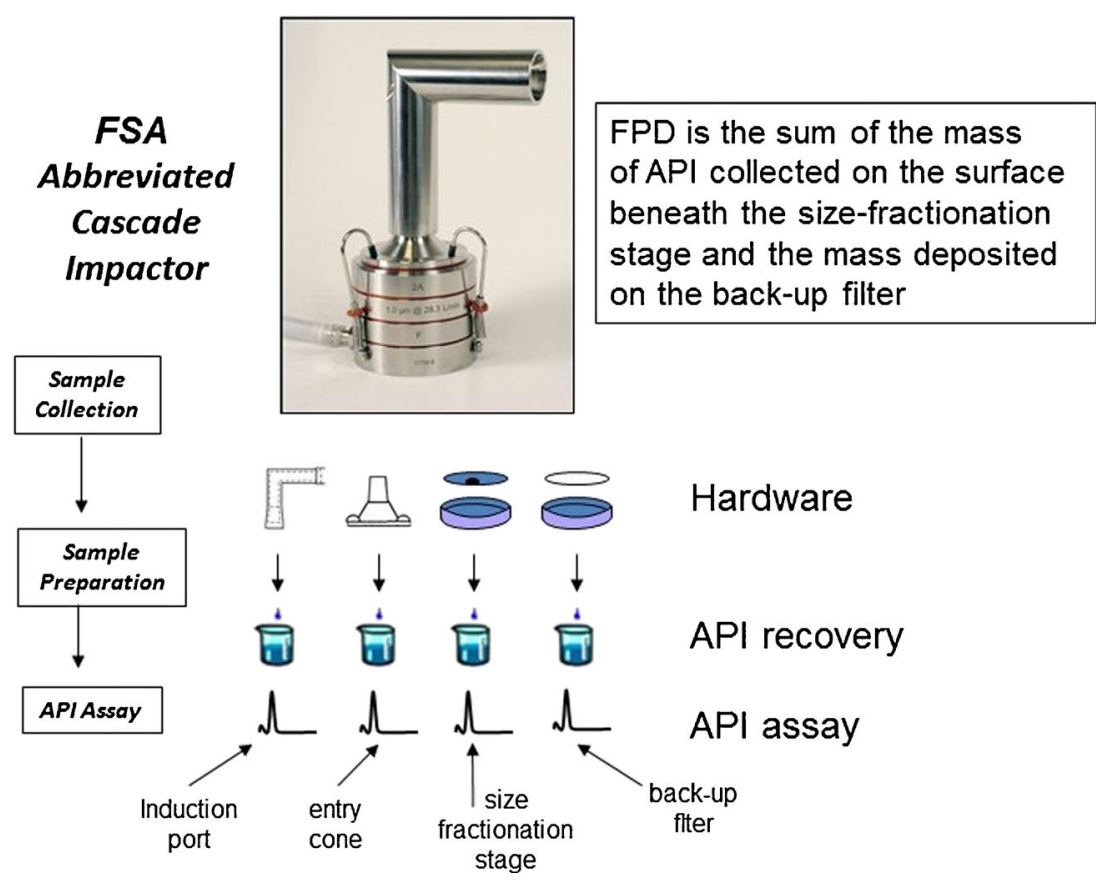

Fig. 1. Key features pertinent to the AIM concept as applied to the control of OIP Quality with a fast screening Andersen (FSA) abbreviated impactor is shown as an example; the non-sizing components, such as the induction port and pre-separator (if used), are retained in the abbreviated apparatus 
understood and validated for the formulation under consideration. The findings reported here do not replace the obligation for the sponsor to undertake appropriate method validation for individual products. It is envisaged that method validation would have to take place when seeking to justify and gain approval from a regulatory agency for the use of an AIMbased method in place of the current pharmacopeial impactor method (13).

\section{MATERIALS AND METHODS}

Five independent laboratories, designated by the code letters "A" through "E," participated in the study which was undertaken in 2013. Five DPI- and two pMDI-delivered products were evaluated (Table I), with the precise composition of each formulation blinded to the study participants to preserve confidentiality and avoid the possibility of bias associated with product choice. Although the DPI type (pre-metered (capsule or blister) or reservoir) and flow resistance were made available by organizations $\mathrm{A}$ and $\mathrm{B}$, organization $\mathrm{D}$ chose not to disclose this information on the grounds that their product was still in development. Two of the DPIs (A and B2) delivered aerosols from combination formulations, whereas the other DPIs (products B1, D1, and D2) each contained a single active component. Organization E evaluated a pMDI-based combination formulation, and organization $\mathrm{C}$ evaluated a one API pMDI but included a VHC to provide additional variety of OIPs that were included. Shot weights ranged from as small as $2 \mathrm{mg} /$ actuation for one of the DPIs evaluated by organization $\mathrm{D}$ to a maximum of $75 \mathrm{mg}$ /actuation for the pMDI assessed by organization $\mathrm{C}$.

A paired study design was used in that each performance metric was determined twice for each of 10 inhalers taken from the same batch, once using the PIM and once using the AIM apparatus. Each inhaler were primed at least 10 times before the first impactor analysis to avoid bias from any dose trend at the beginning of the device. Additional waste actuations between the two impactor analyses was undertaken, if judged necessary by the organization conducting the work, based on their knowledge of their inhaler. Furthermore, the study was balanced by using the AIM apparatus first for five inhalers and the PIM apparatus first for the other five. Each organization used their own validated methods for operating the abbreviated and full resolution CI systems chosen, and for recovery and assay for the API(s) under investigation.
The PIM apparatuses chosen by each organization are listed in Table II. All of the measurements undertaken using the PIM apparatuses followed their appropriate compendial methodologies $(5,6)$. Two organizations (A and D) used a NGI (MSP Corp., St. Paul, MN, USA) operated at 65 and $60 \mathrm{~L} / \mathrm{min}$, respectively. The remainder opted to use the ACI under pharmacopeial flow rate conditions. Organization B tested their DPI at $60 \mathrm{~L} / \mathrm{min}$ with collection stages -1 to 6 in sequence, followed by the back-up filter, in accordance with the recommendations of Nichols for high flow rate testing with this apparatus (14). Organizations $\mathrm{C}$ and $\mathrm{E}$ undertook their measurements with the flow rate at $28.3 \mathrm{~L} / \mathrm{min}$, with the standard stage configuration for pMDI testing (stages 0 to 7 in sequence, followed by the back-up filter). The upper size limit for FPD $_{\text {PIM }}$ was calculated by interpolation, in accordance with the methodology described within monograph 2.9.18 of the Ph.Eur. (5).

The choice of AIM apparatuses is summarized in Table III, with each organization operating their selected system at the same flow rate as that chosen for their corresponding PIM apparatus. A wide variety of options was included; the FSA was used by organizations B, C, and E (Fig. 2a), with organization $\mathrm{C}$ including an empty stage 0 from the full resolution ACI (i.e., the stage without its collection surface immediately beneath) in order to match dead space more closely with that in their unmodified PIM (Fig. 2b). Organization A selected a fast screening impactor (FSI, Fig. 3), and organization D chose to reduce their NGI by relocating the exit filter below stage 3 of the normal configuration (referred to as the "rNGI" configuration (Fig. 4)).

All organizations except B followed the same protocol for each inhaler type. This procedure involved evaluating 10 different inhalers (devices). For five devices, the test order was measurement by PIM followed by AIM apparatus, and for the remaining five devices, the opposite order of testing was followed (Table IV). Organization B investigated only five inhalers of each type, following the test order measurement sequence AIM-PIM-PIM-AIM for each device. Both procedures were attempts to balance the study design for a possible bias in output as a function of individual inhaler use from full towards empty that might otherwise have confounded the results. The protocol restricted the number of analysts to not more than three and required that all determinations (PIM and AIM) for a particular inhaler should be performed by the same analyst (but different analysts could evaluate different inhalers).

Table I. Characteristics of Orally Inhaled Product (OIP) Investigated

\begin{tabular}{|c|c|c|c|c|c|}
\hline Product & Dosage form & Metered/capsule & $\begin{array}{l}\text { Total emitted mass API } \\
+ \text { excipient (mg/actuation) }\end{array}$ & $\begin{array}{l}\text { Flow resistance } \\
\qquad\left(\mathrm{kPa}^{0.5} \mathrm{~min} / \mathrm{L}\right)\end{array}$ & $\begin{array}{l}\text { Number of actuations } \\
\text { to impactor }\end{array}$ \\
\hline A1 & DPI (combination) & Metered & 5 & 0.03 & 6 \\
\hline B1 & DPI & Metered, reservoir & 12 & 0.03 & 1 \\
\hline $\mathrm{B} 2$ & DPI (combination) & Metered, reservoir & 12 & 0.03 & 2 \\
\hline $\mathrm{C} 1$ & $\mathrm{MDI}+\mathrm{VHC}$ & Metered & 75 & & 5 \\
\hline D1 & DPI & Not disclosed & 9 & Not disclosed & 4 \\
\hline D2 & DPI & Not disclosed & 2 & Not disclosed & 5 \\
\hline E1 & MDI (combination) & Metered & 70 & & 10 \\
\hline
\end{tabular}


Table II. Pharmacopeial Impactor Measurement (PIM) Apparatuses Used in the Investigation

\begin{tabular}{lllll}
\hline Organization & Impactor body & Pre-separator & Flow rate (L/min) & Collection surface coating \\
\hline A & NGI & Yes-NGI type & 65 & $\begin{array}{c}\text { Propylene glycol; 10-mL recovery } \\
\text { solvent in pre-separator }\end{array}$ \\
B & ACI (stages -1 to 6 and filter) & Yes-ACI type & 60 & Brij surfactant in glycerol \\
C & ACI (stages 0 to filter) & No & 28.3 & Brij-35 surfactant \\
D & NGI & No & 60 & Brij/glycerol \\
E & ACI (stages 0 to filter) & No & 28.3 & None \\
\hline
\end{tabular}

NGI Next Generation Impactor, ACI Andersen 8-stage non-viable Cascade Impactor

The upper size limit for $\mathrm{FPD}_{\mathrm{AIM}}$ in the reported dataset for all products varied from 4.4 to $5.0 \mu \mathrm{m}$ aerodynamic diameter, depending upon the flow rate and AIM apparatus used (Table III).

\section{Statistical Analysis}

The study was designed based on the information from pilot testing by two organizations, showing that the standard deviation of the ratio AIM/PIM for FPD was likely to be not more than $10 \%$ for any particular product. Thus, a $90 \%$ confidence interval having a width of $10 \%$ was predicted, using the paired design with 10 inhalers and one AIM and one PIM FPD derived from each. This number of inhalers was therefore considered to provide sufficient statistical power to assess whether the use of an AIM apparatus would result in equivalent FPD as when using a PIM apparatus.

Exact equivalence between mean FPD values for a given product obtained by each of the AIM and PIM apparatuses would exist if the ratio $\left[\mathrm{FPD}_{\mathrm{AIM}} / \mathrm{FPD}_{\mathrm{PIM}}\right]$, expressed in percentage terms, is exactly $100 \%$. However, taking into account the finite imprecision of each determination, statistical equivalence was claimed if the $90 \%$ confidence interval of this ratio was contained within the $85-118 \%$ acceptance interval. This criterion is based on a similar specification developed by the European Medicines Agency (EMA) for the assessment of in vitro equivalence for inhaled products (15).

The construction of confidence intervals used the paired design. Firstly, for each inhaler, the ratio between FPDs determined by AIM and PIM was calculated, giving 10 independent ratios for each product. Secondly, the average and standard deviation were calculated, and based on these, a confidence interval was finally determined using the standard statistical technique and normal approximation for these values. The same approach was used for all end-points.

Although determination of FPD was the primary endpoint, the following secondary end-points of comparability between abbreviated and full resolution CI methods were obtained for each apparatus, in which all determinations were reported as amount API (\% target dose) per actuation:

a. Total delivered dose (mass of API) per actuation ex inhaler (DD);

b. Mass of API captured by the induction port, referred to as throat dose (TD) ;

c. Coarse particle dose (mass of API) per actuation (CPD), determined from the relationship: $\mathrm{CPD}=[\mathrm{DD}$ - TD - PS - FPD], where PS $=0$ if no pre-separator was used.

\section{RESULTS}

Table V summarizes the comparison of AIM and PIM for FPD, in terms of the ratio $\left[\mathrm{FPD}_{\mathrm{AIM}} / \mathrm{FPD}_{\mathrm{PIM}}\right]$, of all 10 products investigated. In detail, this table shows the ratio calculated from the mean measures of FPD by AIM and PIM FPD, expressed as a percentage, together with the lower and upper bounds of the associated $90 \%$ confidence interval for assessing equivalence between AIM and PIM. The correlation between FPD, expressed as \% target dose (i.e., label claim dose provided by the participant organization), measured by PIM apparatus (abscissa) and its corresponding value determined by AIM apparatus (ordinate) are shown for each inhaler (Fig. 5).

Table III. Abbreviated Impactor Measurement (AIM) Apparatuses Used in the Investigation

\begin{tabular}{lllclc}
\hline Organization & Impactor body & Pre-separator & Size limit for FPD $(\mu \mathrm{m})$ & Flow rate $(\mathrm{L} / \mathrm{min})$ & Collection surface coating \\
\hline A & FSI & $\begin{array}{c}\text { Y e s - N G I } \\
\text { type }\end{array}$ & 5.0 & 65 & $\begin{array}{c}\text { Coating not required as FPD is } \\
\text { deposited on a filter; 10-mL } \\
\text { recovery solvent in pre- }\end{array}$ \\
& & & & separator \\
B & FSA: stages 1A, 4 + filter & Yes-ACI type & 4.4 & 60 & Brij surfactant in glycerol \\
C & FSA: empty stage 0 & No & 4.7 & 28.3 & Brij-35 surfactant \\
D & rNGI: filter on stage & No & 4.5 & 60 & Trij/glycerol \\
E & F to collect FPD & No & 5.0 & 28.3 & Tween 20 surfactant \\
\hline
\end{tabular}

FPD fine particle dose, FSI fast screening impactor, FSA fast screening Andersen, NGI Next Generation Impactor, $A C I$ Andersen 8-stage nonviable Cascade Impactor, $r N G I$ reduced NGI 
a

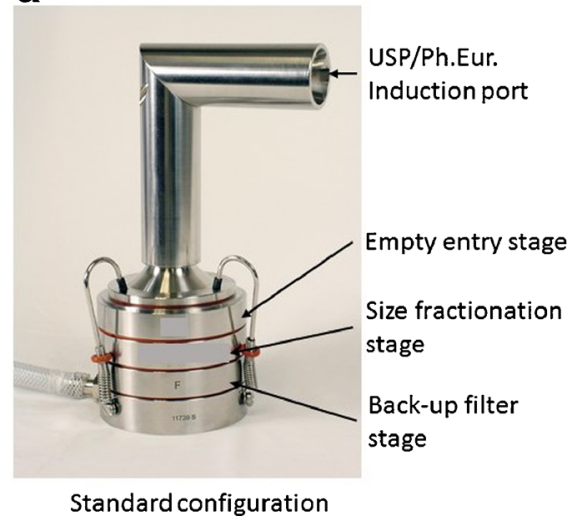

b

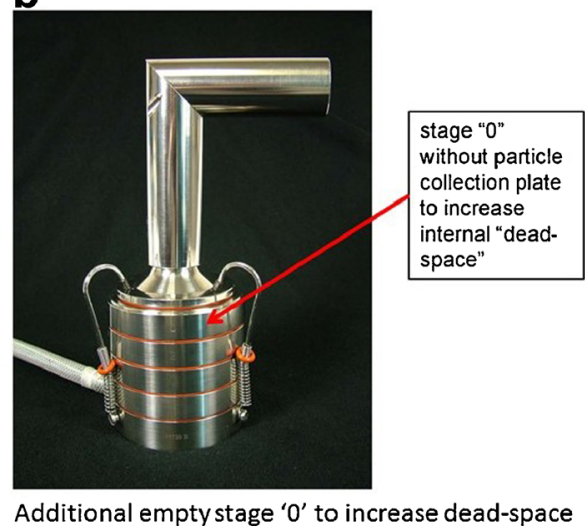

Fig. 2. Fast screening Andersen (FSA) abbreviated impactor configurations: a adopted by organizations B and E; b adopted by organization C

All but one comparison of FPD by AIM apparatus were statistically equivalent to the FPD determined by PIM, in that the $90 \%$ confidence interval for $\left[\mathrm{FPD}_{\mathrm{AIM}} / \mathrm{FPD}_{\mathrm{PIM}}\right]$ was contained within in the $85-118 \%$ acceptance interval. The exceptions was the data for combination product A1a, where FPD $_{\text {AIM }}$ was about $20 \%$ greater than the corresponding

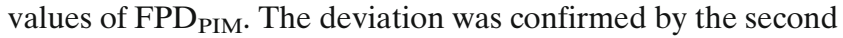
component of the combination (A1b), which also had a slightly off-target FPD ratio, although in this case equivalence was fulfilled. Product A1 was the sole example in this study that was investigated using the FSI-NGI pair of apparatuses. Looking at the overall average for all the products evaluated, $\left[\mathrm{FPD}_{\mathrm{AIM}} / \mathrm{FPD}_{\mathrm{PIM}}\right]$ was $104.6 \%$. This outcome was within the allowance of $5 \%$ as potential bias arising from API deposited on internal surfaces of the compendial full resolution CIs $(5,6)$, given that losses are likely to be greater with the increased internal surfaces associated with the PIM apparatuses, and will, therefore, tend to bias the ratio $\left[\mathrm{FPD}_{\mathrm{AIM}} / \mathrm{FPD}_{\mathrm{PIM}}\right]$ to be greater than the ideal value of $100 \%$. Correlations between individual measures of FPD on an inhaler-by-inhaler basis were moderately good, but inevitably some scatter was evident, so that the overall correlation coefficient $\left(r^{2}\right)$ for the complete dataset was 0.885 .

The overall mean ratios for AIM/PIM associated with each of the secondary end-point measures DD and TD were both $102 \%$, and therefore close to the ideal value of $100 \%$; the scatter of the individual values of these ratios (Fig. 6) ranged from 97.0 (TD, product $\mathrm{B} 2 \mathrm{~b}$ ) to 106.7 (TD, product A1b). Their associated $90 \%$ confidence intervals were within the $85-118 \%$ acceptance interval set for FPD for 19 out of 20
AIM/PIM comparisons (Fig. 7). The notable exception was product $\mathrm{C} 1$, whose confidence interval length was from 88.1 to $125.9 \%$ for TD, but only from 99.5 to $102.9 \%$ for DD. Mean values of the end-point, CPD, across all products had greater associated variability than was observed with the other secondary metrics (Fig. 6). This behavior resulted in a slightly lower overall mean ratio at $95 \%$ with increased range of individual results between products ( 70 to $123 \%$ ). The associated $90 \%$ confidence intervals reported for CPD spanned the range 67 to $130 \%$ when taken together (Fig. 7), and as a consequence, several individual results were wider than the acceptance interval chosen for FPD.

\section{DISCUSSION}

An initial attempt to validate equivalence of the AIM concept prior to the present investigation was undertaken in a highly controlled environment with a canister of a single pMDI-delivered formulation (salbutamol sulfate) from the same lot manufactured within a short time of each other (16). The outcome demonstrated good agreement between abbreviated and full-resolution CI measurements of therapeutically important properties, in particular FPD, based on an upper size limit of $4.7 \mu \mathrm{m}$ aerodynamic diameter. A series of subsequent comparisons undertaken by a variety of organizations involved with inhaler testing, using a wide range of formulations and inhaler types (pMDI, DPI, nebulizer), confirmed good agreement could be achieved between FPD (whatever the upper size limit) determined by AIM and full
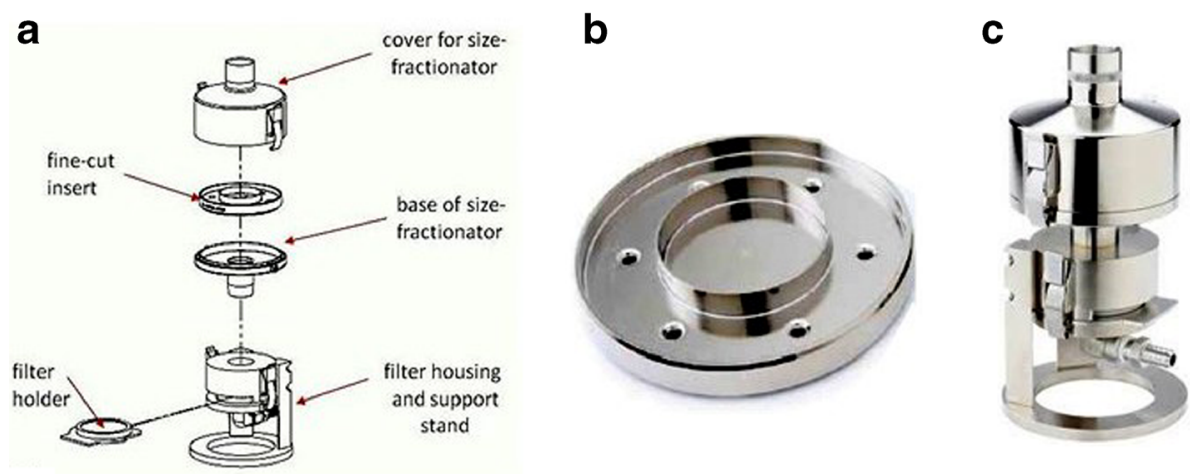

Fig. 3. Fast screening impactor (FSI) adopted by organization A; a schematic of assembly; b size fractionating stage; c assembled apparatus 


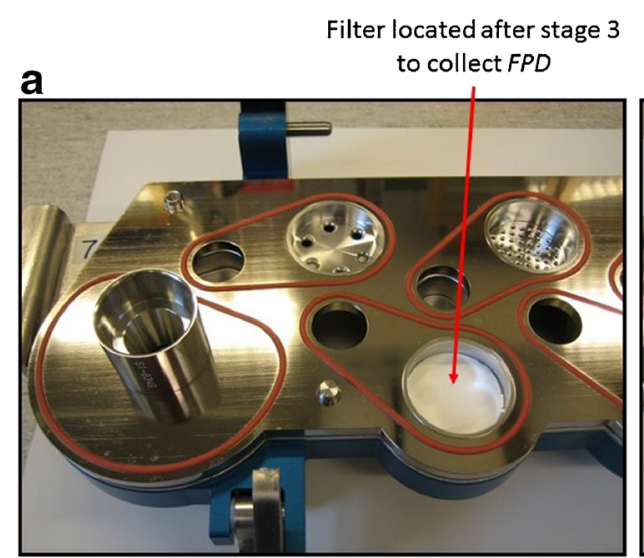

Filter location

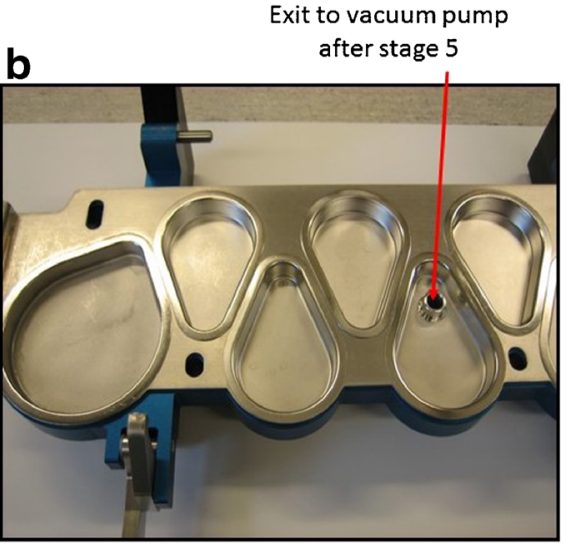

Exit to vacuum source

Fig. 4. Reduced NGI (rNGI) configuration adopted by organization D

resolution CIs, provided that certain provisions are made (17). These precautions are as follows:

1. Ideally, the abbreviated impactor should be related to the "parent" full-resolution CI. For example, the FSA is ideally paired with the Andersen 8-stage non-viable CI, since both have similar inter-stage dead-space by virtue of using flat collection plates. The pairing of the FSA, having flat collection plates, with the fullresolution Andersen viable CI that utilizes Petri dishes as the collection surface would therefore not be as appropriate a match of apparatuses (17);

2. The user who is considering to choose the FSI that was developed specifically for rapid screening of formulations in early stage development, but which has no "parent" full resolution CI, should appreciate that extra care may be needed to ascertain that important properties such as internal dead space upstream of the size-fractionating stage of the FSI match as closely as possible with that of the selected full resolution CI;

3. Precautions are required to avoid particle bounce on the collection surface for the size-fractionated particles (18). This consideration is especially pertinent for all types of AIM apparatus. Here, the particle size distribution presented to the size-fractionating stage will likely contain a greater proportion of larger particles than would be the case for the same stage comprising part of a PIM apparatus, in which preceding stages act to remove much of the coarser particle fraction. Care is also needed to avoid the physical displacement of the stage coating by the high velocity air flow emerging from the nozzles of the stage at which sizefractionation takes place (19).

Table IV. Study Design Based on 10 Inhalers of the Same Product

\begin{tabular}{lll}
\hline Devices & First FPD determination & Second FPD determination \\
\hline $1-5$ & AIM & PIM \\
$6-10$ & PIM & AIM \\
\hline
\end{tabular}

$F P D$ fine particle dose, AIM abbreviated impactor measurement, $P I M$ pharmaceutical impactor measurement
4. Dead-space matching needs to be undertaken. This precaution is especially important if the product being tested is a DPI $(20,21)$ where the compendial test method involves controlling flow acceleration (rise time), thus creating a well-defined flow rate-time profile that is required to operate the inhaler $(5,6)$. The same precaution is also necessary if a pMDI-based formulation containing low volatile excipients such as ethanol is being evaluated (22). In this context, it is worthwhile noting that the rNGI approach avoids the need for dead space matching, if the flow exiting the back-up filter is returned to the impactor rather than being extracted directly to the vacuum source, as was done by participant D (Fig. 4).

5. The FSI was calibrated with particles of known aerodynamic size (23). However, to the best knowledge of the authors, it has thus far been tacitly assumed for the various FSA-based impactors that the stage cut-point at a given flow rate of that particular AIM apparatus is identical with that of the equivalent stage of the full resolution ACI. Even if the stage used in the FSA has been taken from an ACI, in a typical determination of FPD, the particle size distribution upstream will be different to that encountered in the full resolution impactor (see consideration 3). The risk of a bias in FPD arising from such a cause is believed to be small, given that the nozzle dimensions and jet-to-plate distance largely determine the stage cut-point size (24) and neither change comparing the AIM and PIM configurations. Nevertheless, there would be merit in undertaking a formal evaluation in the future with the objective of providing confirmatory evidence that this assumption is valid.

Examining these potential causes of measurement bias in the light of methodologies that were adopted for the present study, AIM-to-PIM apparatus pairing (compare Tables II and III) was undertaken where this was possible (organizations B, $\mathrm{C}$, and E). Organization D used a reduced version of the NGI as their choice of AIM apparatus, thereby preserving identical dead-space between abbreviated and full resolution systems. Only organization A, who used the FSI as their AIM apparatus, had an unrelated internal geometry to that of their full resolution NGI as their choice of PIM system. Organizations 
Table V. Summary of Evaluation for AIM/PIM Equivalence in FPD for the Orally Inhaled Products Investigated

\begin{tabular}{llccc}
\hline Organization code & Product code & Mean [FPD AIM $/ F_{\text {PIM }}$ (\%) & $\begin{array}{c}\text { Lower bound 90\% } \\
\text { confidence interval (\%) }\end{array}$ & $\begin{array}{c}\text { Upper bound 90\% } \\
\text { confidence interval (\%) }\end{array}$ \\
\hline A & A1a & 120.1 & 116.3 & 123.9 \\
B & A1b & 114.1 & 110.9 & 117.2 \\
& B1 & 98.8 & 95.9 & 101.8 \\
& B2a & 110.8 & 106.8 & 114.8 \\
C & B2b & 107.5 & 103.6 & 111.4 \\
D & D1 & 101.9 & 96.9 & 106.9 \\
& D2 & 102.2 & 101.1 & 103.4 \\
E & E1a & 89.5 & 85.9 & 93.2 \\
& E1b & 100.3 & 95.8 & 104.8 \\
\end{tabular}

FPD fine particle dose

B to D took precautions to minimize bias from particle bounce by choosing to pre-coat collection surfaces in both AIM and PIM apparatuses (Tables II and III). Organization A used a FSI as their AIM-based apparatus. This equipment collects the size-fractionated portion of the dose onto a filter, so there was no need for a coating. They did, however, place $10-\mathrm{mL}$ of API recovery solvent in the cup at the center of the size fractionating stage to avoid coarse particle bounce at this location. The underlying cause of the higher than anticipated values of FPD AIM $_{\text {for each component of their product }}$ (Table V) could therefore not be attributed to an obvious cause, such as particle bounce. Organization D also coated the collection surfaces of both PIM and AIM impactors, and reported good agreement for one of their DPIs (D1). However, FPD determined by their AIM apparatus for their second product (D2) was somewhat lower than that obtained using their PIM system, suggesting that an alternative process not readily apparent in terms of the precautions outlined above may have been responsible for that particular outcome. Another factor pertinent to all DPIs evaluated that may have contributed to the observed outcomes in Table $\mathrm{V}$ could be small differences in the flow rate-time profiles that were achieved during evaluation for each device type in this OIP class. However, this study was not intended to identify each underlying cause for the average difference between each set of FPD results by PIM and AIM systems, since it is recognized that such investigations could likely involve proprietary formulation and/or device-specific issues that were not shared within the study collaboration. In any case, such detailed considerations should form part of the due diligence associated with the quest to adopt an AIM-based approach on a product-by-product basis, as a key component of the method development and validation processes. Finally, small deviations between the upper size limits chosen for $\mathrm{FPD}_{\mathrm{AIM}}$, present in data from participants $\mathrm{B}, \mathrm{C}$, and $\mathrm{D}$, that were always finer than the $5.0 \mu \mathrm{m}$ aerodynamic diameter limit specified in the Ph.Eur. (5)] (Table III), might have been expected to result in slightly, but consistently reduced values of FPD $_{\mathrm{AIM}}$

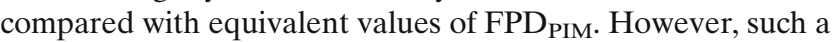
trend was not evident in the data for products B1, B2, C, D1,

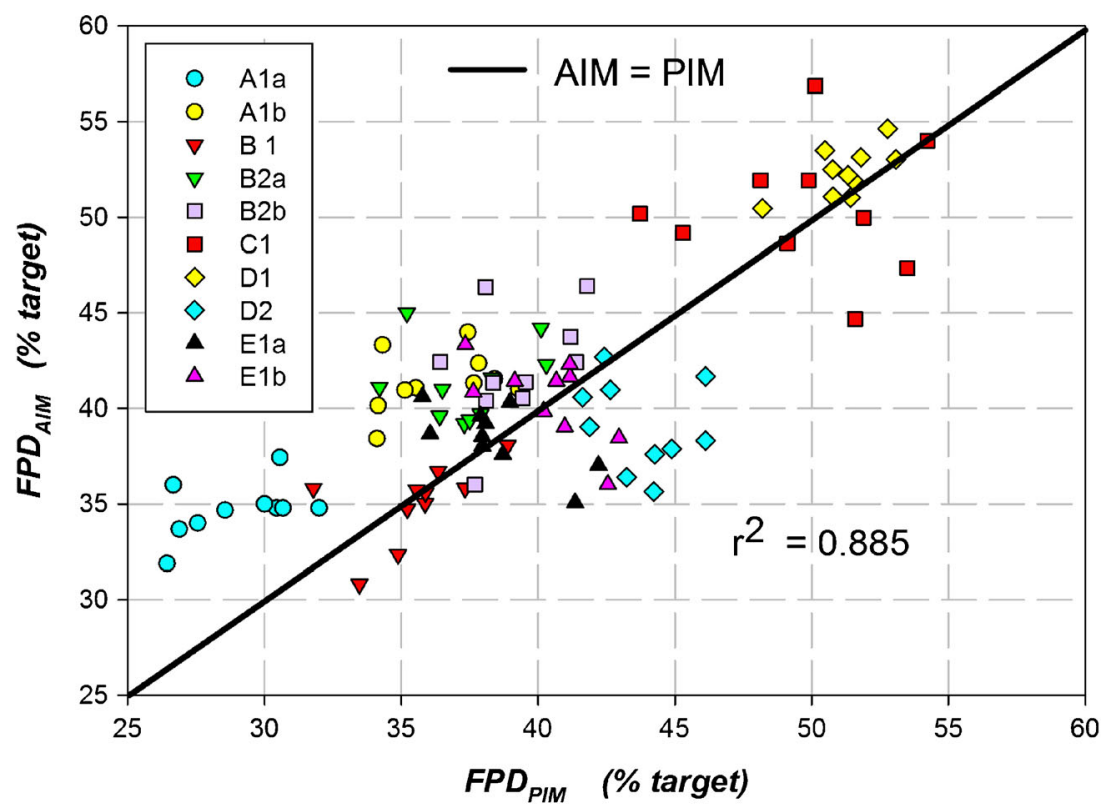

Fig. 5. Correlation between AIM and PIM FPD, expressed as a percentage of the target dose per actuation 


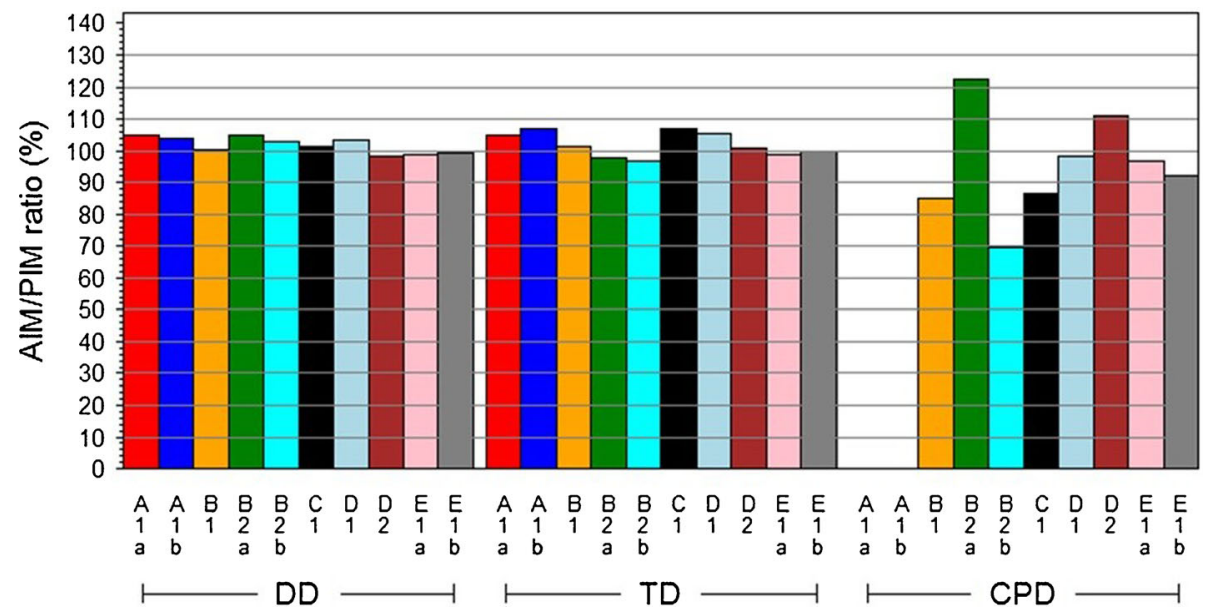

Fig. 6. Individual product means for secondary metrics DD, TD, and CPD

and D2, shown in Fig. 5. In summary, it is likely that a combination of some or all of these factors was contributory to the small discrepancies that were observed between PIM- and AIM-derived measures for each OIP.

Looking at the data obtained by the two organizations $(\mathrm{C}$ and $\mathrm{E}$ ) that evaluated pMDIs, the choice by one of them (organization $\mathrm{C}$ ) to incorporate a VHC add-on device with their inhaler did not bias FPD $_{\text {AIM }}$ from the line of identity with FPD $_{\text {PIM }}$, based on a visual comparison with the corresponding data obtained with the other pMDI that was evaluated. These measurements were undertaken without any delay between inhaler actuation and sampling, even though a VHC was present, as the purpose was to compare AIM- and PIM-based methodologies, rather than to explore VHC performance per se. Furthermore, there were no differences in pMDI-VHC configuration between the measurements undertaken with the abbreviated and full resolution impactor. However, it is apparent from visual inspection of the results for the individual inhaler/VHC combinations (Fig. 5) that the variability of that data set was slightly greater than that from organization E. Caution should be exercised in drawing a firm conclusion concerning this particular aspect, since this study was neither designed nor intended to investigate directly this potential influence on relative variability (i.e., by evaluating the same pMDI product with and without VHC being present).
Before considering the differences between AIM- and PIM-derived values of the metrics of interest in the present study in more detail, it is important to note that the pharmacopeial compendia permit up to a $5 \%$ lower value for the total recovered mass of API from a PIM system as compared to the standard delivered dose determination by Dosage Unit Sampling Apparatus (DUSA) (5,6). This latitude for discrepancy is provided to account for losses of medication to the internal surfaces (e.g., walls of stage metalwork) from which any deposited particulate is not normally recovered. In the ACI and likely in its associated AIM alternatives, such losses are greatest with the largest particles whose motion in the air flow streamlines through the impactor is most influenced by inertial effects and gravity (25). Any bias introduced by stage removal would therefore be expected to be most apparent upstream of the impaction stage separating coarse from fine particle fractions. Furthermore, the reduced internal surface area available for particle losses to occur, associated with removing upper stages with the FSA configurations used by organizations $\mathrm{B}, \mathrm{C}$, and $\mathrm{E}$ (Table II), would therefore contribute to mean values of the ratio $\left[\mathrm{FPD}_{\mathrm{AIM}} / \mathrm{FPD}_{\mathrm{PIM}}\right]>100 \%$, and this outcome is consistent to a greater or reduced extent for the data from products B2, C, and E (Table V). However, the results for product $\mathrm{B} 1$ are the exception, with the mean value of this ratio at $98.8 \%$. Even so, the deviation from

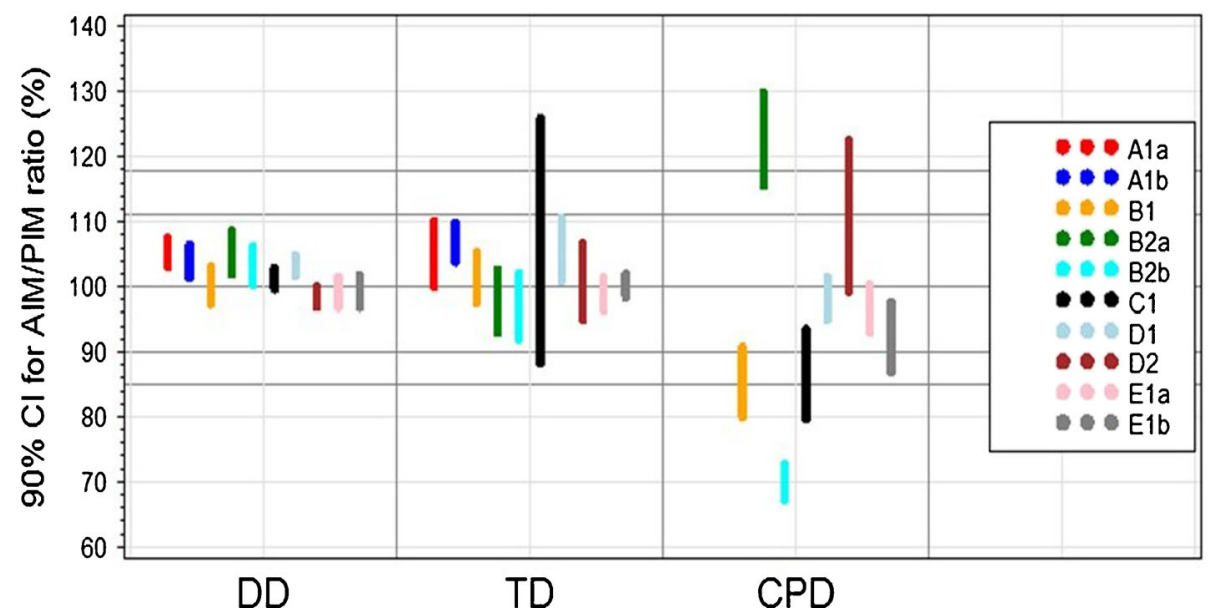

Fig. 7. $90 \%$ Confidence intervals for AIM/PIM ratio of secondary metrics DD, TD, and CPD for each product 
ideal matching of AIM- and PIM-derived measures is so small that it can probably be accounted for by the random variability associated with impactor-based measurements by either type of apparatus. Organization A used an FSI for their AIM-based measurements, whose internal geometry is quite different to that for the NGI, being based on the shape of the pre-separator for this PIM apparatus (23). Interestingly, they reported mean values of $\left[\mathrm{FPD}_{\mathrm{AIM}} /\right.$ FPD $_{\text {PIM }}$ ( Table V) that were significantly higher than $100 \%$ for both products A1a (120.1\%) and to a lesser extent A1b (114.1\%). These deviations may have originated from differences in particle deposition behavior between their particular choices of AIM and PIM apparatuses. However, other organizations using this pairing of systems have previously reported better agreement with DPI products, after taking precautions to match dead-space of the FSI with that of the NGI (21) and separately also with pMDI products (26). The underlying cause for the observed behavior of the combination DPI from this participant may therefore be product- rather than apparatus-related. Moreover, further investigations by organization A to find a root cause of their deviations have not so far revealed a definitive explanation. Finally, organization D, who used a reduced NGI option to create their AIM-based apparatus, so that dead-space matching could be more easily maintained with the full resolution NGI, reported mean $\left[\mathrm{FPD}_{\mathrm{AIM}} / \mathrm{FPD}_{\mathrm{PIM}}\right]$ values of 102.2 and $89.5 \%$ for products $\mathrm{D} 1$ and $\mathrm{D} 2$, respectively (Table V). The former outcome was sufficiently close to the ideal match that random variability could account for the observed deviation. However, the latter result, though remaining just within the acceptance criterion based on its associated $90 \%$ confidence interval ( 85.9 to $93.2 \%$ ), was lower than expected, and further investigation has not resulted in establishing a definitive cause. In summary, given the variety of outcomes observed across all participating organizations, the best advice for the sponsor proposing to make use of this combination of test apparatuses is to evaluate matching of FPD (and any other metrics of interest) on a product-by-product basis before selecting the AIM apparatus to use.

The overall mean AIM/PIM ratios of the secondary metrics DD and TD (102\% in both cases) based on all the products evaluated were close to ideal (Fig. 6), with small associated confidence intervals well within the acceptance range from 85 to $118 \%$ for all products, except for measures of $\mathrm{TD}$ with product $\mathrm{C} 1$ (Fig. 7), where the confidence interval ( 88.1 to $125.9 \%$ ) was significantly wider than the $85-118 \%$ acceptance range, formally applicable only for the assessment of FPD, but used as a convenient benchmark for these secondary metrics. The fact that the associated variability for DD with this product (99.5 to $102.9 \%$ ) was much smaller and close to the equivalent values for the other products can be explained by the fact that the VHC used in the evaluation of product $\mathrm{C} 1$ removed almost all the TD, without affecting the magnitude of DD. This outcome links well with the fact that values of CPD were close to zero $(<5 \%$ target dose for six products and $<10 \%$ for the other two products). Although the overall mean AIM/PIM ratio for this metric $(95 \%)$ was quite close to ideal, the span of all reported $90 \%$ confidence intervals encompassed a wider than ideal range from 67.1 to $129.7 \%$, indicating significantly greater variability compared with the other measures. That said, it is unlikely that AIM- based methods would be used to assess CPD on its own, since the focus of impactor-based measurements is to establish FPD, given the importance of the latter measure in quantifying the efficacy of the drug product delivered by a particular OIP to the lungs.

\section{CONCLUSIONS}

Nine out of 10 data sets collected in a designed experiment comparing AIM with PIM apparatuses for the assessment of the primary measure, FPD, fulfilled the equivalence acceptance criteria defined before the investigation. Based on all 10 products, the FPD determined by an AIM-based technique was on average $5 \%$ greater than the equivalent measure made by a PIM apparatus. The findings from this investigation provide good support for statistical equivalence of AIM and European Pharmacopoeia PIM for determination of this physiologically important measure across a wide range of OIP platforms and measurement techniques. It is concluded that an AIM-based approach has the potential of being used as an alternative to determine FPD as the critical quality attribute for OIP aerosol aerodynamic particle size in the current European Pharmacopeial method. However, the validation of an AIM-based technique as a viable alternative to the PIM approach should be undertaken on a product-by-product basis. Even if there is an offset between AIM- and PIM-determined FPD, as seen with some of the products in this study, the AIMbased method may still be viable if the cause of the offset is investigated and validated. Good impactor practice would require a supporting validation package on a product by product basis, in order to justify the choice of brand and associated methodology when using AIM for the determination of FPD.

\section{ACKNOWLEDGMENTS}

The authors wish to thank their colleagues Rubina Ali, Caroline Ahlm Persson, Regina Buechel, Cathy Doyle, Benjamin Ehrlein, Mark Nagel, Sandra Schroeder, Magdalena Thews, and Michael Zier, as well as other members of the EPAG Impactor Sub-Team, who provided support in the design and execution of this work. The organizations employing the authors provided funding and resources to complete the study.

\section{REFERENCES}

1. Heyder J, Svartengren MU. Basic principles of particle behavior in the human respiratory tract. In: Bisgaard H, O'Callaghan C, Smaldone GC, editors. Drug delivery to the lung. New York: Marcel Dekker; 2002. p. 21-45.

2. Labiris NR, Dolovich MB. Pulmonary drug delivery. Part I: physiological factors affecting therapeutic effectiveness of aerosolized medications. Br J Clin Pharmacol. 2003;56(6):588-99.

3. Patton JS, Fishburn S, Weers J. The lungs as a portal of entry for systemic drug delivery. Proc Am Thoracic Soc. 2004;1:338-44.

4. Tougas TP, Christopher D, Mitchell J, Lyapustina S, Van Oort M, Bauer R, et al. Product lifecycle approach to cascade impaction measurements. AAPS PharmSciTech. 2011;12(1):312-22.

5. European Directorate for Quality in Medicines (EDQM). European pharmacopeia 8.0, monograph 2.9.18. Preparations for 
inhalations: aerodynamic assessment of fine particles. Strasburg, France EDQM 2014 (January).

6. US Pharmacopeial Convention. United States Pharmacopeia 38/ National Formulary 33, Chapter $<601>$ Aerosols, nasal sprays, metered-dose inhalers, and dry powder inhalers. Rockville, MD, 2015.

7. Bonam M, Christopher D, Cipolla D, Donovan B, Goodwin D, Holmes $\mathrm{S}$, et al. Minimizing variability of cascade impaction measurements in inhalers and nebulizers. AAPS PharmSciTech. 2008;9(2):404-13.

8. Christopher D, Curry P, Doub W, Furnkranz K, Lavery M, Lin K, et al. Considerations for the development and practice of cascade impaction testing including a mass balance failure investigation tree. J Aerosol Med. 2003;16(3):235-47.

9. Marple VA, Roberts DL, Romay FJ, Miller NC, Truman KG, Van Oort M, et al. Next generation pharmaceutical impactor. Part 1: design. J Aerosol Med. 2003;16(3):283-99.

10. Mitchell JP, Tougas T, Christopher JD, Lyapustina S, Glaab V. (2012) The abbreviated impactor measurement and efficient data analysis concepts: why use them and when. In: Dalby RN, Byron PR, Peart J, Suman JD, Young PM, editors. Respiratory drug delivery 2012. River Grove, IL, USA: Davis Healthcare International; 2012. p. 731-736.

11. Tougas T, Mitchell J, Morgan B, Strickland H. Introduction. In: Tougas TP, Mitchell JP, Lyapustina SL, editors. Orally inhaled products: good cascade impaction practices, AIM and EDA. New York: Springer; 2013. p. 1-14.

12. Nichols S, Mitchell J. Impactor use survey 2012: an industry perspective on behalf of the European Pharmaceutical Aerosol Group (EPAG). Drug Delivery to the Lungs 23, The Aerosol Society, Edinburgh, UK; 2012. pp. 216-219.

13. Nichols SC, Mitchell JP, Tougas TP, Christopher JD, Holmes S. The regulatory and compendial pathways to acceptance for AIM and EDA concepts. In: Tougas TP, Mitchell JP, Lyapustina SL, editors. Orally inhaled products: good cascade impaction practices, AIM and EDA. NY: Springer; 2013. p. 359-74.

14. Nichols SC, Brown DR, Smurthwaite M. New concept for the variable flow rate Andersen cascade impactor and calibration data. J Aerosol Med. 1998;11(S1):133-8.

15. European Medicines Agency (EMA): Requirements for clinical documentation for orally inhaled products (OIP) including the requirements for demonstration of therapeutic equivalence between two inhaled products for use in the treatment of asthma and chronic obstructive pulmonary disease (COPD) in adults and for use in the treatment of asthma in children and adolescents. London, UK. CPMP/EWP/4151/00 Rev. 1, 2009. Available at URL: http://www.ema.europa.eu/docs/en_GB/document_library/ Scientific_guideline/2009/09/WC500003504.pdf ; visited June 1st, 2015.

16. Mitchell JP, Nagel MW, Doyle C, Ali RS, Avvakoumova V, Christopher D et al. Relative precision of inhaler aerodynamic particle size distribution (APSD) metrics by full resolution and abbreviated Andersen Cascade Impactors (ACIs): accuracy and precision study of inhaler aerodynamic particle size distribution
(APSD) metrics by full resolution and abbreviated Andersen cascade impactors (ACIs). Part 1-Investigation of bias in extrafine mass fraction with AIM-HRT impactor. AAPS PharmSciTech, 2010;11(2):843-851.

17. Copley M, Mitchell JP, Svensson M, Christopher D, Quiroz J, Daniels G, et al. Validating AIM-based instrumentation and associated measurement techniques. In: Tougas TP, Mitchell JP, Lyapustina SL, editors. Good cascade impaction practices, AIM and EDA. New York: Springer; 2013. p. 285-359.

18. Mitchell JP, Nagel MW, Avvakoumova V, MacKay H, Ali R. The Abbreviated Impactor Measurement (AIM) concept: part I-influence of particle bounce and re entrainment-evaluation with a "dry" pressurized metered dose inhaler (pMDI) based formulation. AAPS PharmSciTech. 2009;10(1):243-51.

19. Mitchell JP, Nagel MW, Doyle C, Ali RS, Avvakoumova V, Christopher D, et al. Relative precision of inhaler aerodynamic particle size distribution (APSD) metrics by full resolution and abbreviated Andersen Cascade Impactors (ACIs): part 2-investigation of bias in extra-fine mass fraction with AIM-HRT impactor. AAPS PharmSciTech. 2010;11(3):1115-8.

20. Keegan GM, Lewis DA. Rapid prototype screening with the Copley Fast Screening Andersen (FSA). In: Dalby RN, Byron PR, Peart J, Suman JD, Young PM, editors. Respiratory Drug Delivery 2012. River Grove: Davis Healthcare International; 2012. p. 469-72.

21. Pantelides PN, Bogard H, Russell-Graham D, Cooper AD, Pitcairn GR. Investigation into the use of the Fast Screening Impactor as an abbreviated impactor measurement (AIM) tool for dry powder inhalers. In: Dalby RN, Byron PR, Peart J, Suman JD, Young PM, editors. Respiratory Drug DeliveryEurope 2011. River Grove: Davis Healthcare International; 2011. p. 391-5.

22. Mitchell JP, Nagel MW, Avvakoumova V, MacKay H, Ali R. The abbreviated impactor measurement (AIM) concept: part 2-influence of evaporation of a volatile component-evaluation with a "droplet producing" pressurized metered dose inhaler (pMDI)based formulation containing ethanol as co-solvent. AAPS PharmSci Technol. 2009;10(1):252-7.

23. Roberts DL, Romay F. Design of the fast screening impactor based on the NGI pre-separator. Drug Delivery to the Lungs20, The Aerosol Society, Edinburgh, UK, 2009; 20:206-209. Available at: http://ddl-conference.org.uk/ index.php?q=previous_conferences. Accessed 2 Jul 2015.

24. Marple VA, Liu BYH. Characteristics of laminar jet impactors. Environ Sci Technol. 1974;8(7):648-54.

25. Mitchell JP, Costa PA, Waters S. An assessment of an Andersen Mark-II cascade impactor. J Aerosol Sci. 1987;19(2):21321.

26. Sheng G, Watanabe W (2010) Feasibility of fast screening impactor as a screening tool. Drug Delivery to the Lungs-21, The Aerosol Society, Edinburgh, UK, 2010;21:390-393. http://ddlconference.org.uk/index.php?q=previous_conferences. Accessed 2 Jul 2015. 